\title{
The Use of Antidepressants in the Long-Term Treatment Should not Im- prove the Impact of Fibromyalgia on Quality of Life
}

\author{
Carta $\mathrm{MG}^{1, *}$, Ruggiero $\mathrm{V}^{2}$, Sancassiani $\mathrm{F}^{1}$, Cutrano $\mathrm{F}^{1}$, Manca $\mathrm{AR}^{1}$, Peri $\mathrm{M}^{3}$, Fais $\mathrm{A}^{4}$ and Cacace $\mathrm{E}^{2}$ \\ ${ }^{I}$ Dipartimento di Sanità Pubblica e Medicina Clinica e Sperimentale, Università di Cagliari e Center of Liaison \\ Psychiatry and Psychosomatics, University Hospital of Cagliari, Italy \\ ${ }^{2}$ Dipartimento di Scienze Mediche "Mario Aresu”- Universita' degli Studi di Cagliari-09042 Monserrato - Cagliari, Italy \\ ${ }^{3}$ Dipartimento di Scienze Chirurgiche - Universita' degli Studi di Cagliari- 09042 Monserrato - Cagliari, Italy \\ ${ }^{4}$ Dipartimento di Scienze della Vita e dell'Ambiente, Università di Cagliari, 09042 Monserrato, Cagliari, Italy
}

\begin{abstract}
Background: Antidepressant (AD) drugs are effective in the short term treatment of fibromyalgia (FM). It may be useful to study the long-term impact of AD on patients with FM.

Methods: One-year follow-up study on 23 females with FM divided into groups on AD (ADg-N=7), and not taking AD $(\mathrm{NADg}-\mathrm{N}=11$ ). Evaluation at $\mathrm{t} 1$ and at the end ( $\mathrm{t} 2)$ with the Fibromyalgia Impact Questionnaire (FIQ); at t2 with: SCIDIV; Mood Disorder Questionnaire (MDQ); Short Form-12; Hamilton Depression Rating Scale (HAM-D); Functioning Assessment Short Test (FAST)

Results: After a year the AD group showed a worst impact of the disease by FIQ ( $\mathrm{p}=0.017$ ), worsened quality of life by SF-12 ( $<<0.01)$, and disability linked to bipolar symptoms by FAST $(\mathrm{p}=0.05)$. About $40 \%$ of the sample was screened positive at MDQ without difference in the two groups. The patients who recovered from a depressive episode did not differ between ADg and NADg (20\% vs 33.3\%), and were fewer than expected from the literature (40-60\%). The HAM-D score at the end of the trial was worse in the $\operatorname{ADg}(\mathrm{p}<0.03)$.

Limitations: Observational research on few patients, not specifically designed to test the hypothesis. The results have a heuristic value only.

Discussion: The results should be read in the light of the high prevalence of patients screened positive for Bipolar Disorders and of the well-known poor response of the mood symptoms to antidepressants in Bipolar Depression. The deterioration in the long-term management of FM patients following AD treatments suggests the need for new and robust studies.
\end{abstract}

Keywords: Fibromyalgia, Antidepressants, Efficacy, Long-Term Treatment, Quality of life.

\section{INTRODUCTION}

Clinical studies have revealed the efficacy of antidepressant drugs in the treatment of fibromyalgia (FM) [1]. Antidepressants, SNRI in particular, are commonly used in the current treatment of FM even though a recent meta-analysis shows that SNRI duloxetine and milnacipran produced a small incremental benefit over placebo in reducing pain, were similar to placebo against sleep problems, showed no substantial superiority over placebo in reducing fatigue and improving the Quality of Life, and had higher dropout rates than placebo due to their adverse effects [2].

The randomized controlled clinical trials have provided information on short-term treatment. It may be useful therefore to check also the long-term impact of treatment with antidepressants on patients with FM. This is all the more

\footnotetext{
* Address correspondence to this author at the Department of Public Health and Clinical and Molecular Medicine, University of Cagliari. Italy, Via Ospedale. 09123 Cagliari Italy; Tel +39 335499994

Fax: +39, 070 6093498; E-mail: mgcarta@tiscali.it
}

important considering that a high frequency of manic symptoms was reported in patients with FM, who were supposed to be at risk of bipolar disorders [3]. The diagnosis of bipolar disorders is very difficult even for the specialist psychiatrist thus hypothetically some fibromyalgia patients treated with antidepressants may suffer from or may be vulnerable to bipolar disorders. The antidepressant treatment without stabilizers in the course of a bipolar disorder should be ineffective against the depressive symptoms and can induce manic switch [4], mixed symptoms [5] and rapid cycling course [4]. These negative effects and their consequences on the life of the patient do not always become manifest in the short term.

\section{OBJECTIVE}

To measure the change in the overall impact of FM in a cohort of consecutive women with Fibromyalgia attending a Consultancy Rheumatologic Service, and to measure the difference of the impact of the illness on dimensions as function, pain level, fatigue, sleep disturbance, psychological distress [6] by treatment (antidepressants vs no antidepressants - independent variable). 
Table 1. Clinical Characteristics of the Sample at t 2

\begin{tabular}{|c|c|c|c|c|}
\hline & Antidepressant & No Antidepressants & Statistics ANOVA \\
(df 1,16,17)
\end{tabular}

\section{METHODS}

\section{Design}

One-year naturalistic follow-up study. The patient sample was evaluated in 2011 (t1) and 12 months later (t2); throughout the follow up the patients who completed the study were followed by the Consultancy Rheumatologic Service at the Azienda Ospedaliera Universitaria of Cagliari with no less than 4 visits in the year.

\section{Sample}

23 females affected by FMS were enrolled. All FMS patients were examined by a qualified rheumatologist and fulfilled the American College of Rheumatology Diagnostic Criteria [7]. The sample was divided at the end of the followup into two groups on the basis of being or not on antidepressant therapy during the follow up at the minimal therapeutic dosage for at least15 days.

\section{Study Tools}

At $\mathrm{t} 1$ and $\mathrm{t} 2$ the subjects filled in the Fibromyalgia Impact Questionnaire (FIQ) [8] in the Italian version [9]. The FIQ is an instrument designed to measure the overall impact of fibromyalgia over many dimensions (function, pain level, fatigue, sleep disturbance, psychological distress, etc.). Its scores range from 0 to 100 , with 100 marking the worst case.

At $t 1$ the following instruments were also administered by a trained psychologist or psychiatrist:

- the DSM-IV SCID-CV interview to assess the presence of lifetime and current psychiatric disorders [10];

- the Mood Disorder Questionnaire (MDQ) [11] in the Italian version [12] for the assessment of bipolar spectrum disorders;
- the Short Form Health Survey to evaluate the Quality of life (SF-12) [13]. The SF-12 includes the following dimensions: physical activity, physical health limitations on role or activities, emotional state, physical pain, self-evaluation of general state of health, vitality, social activity and mental health. The period of measurement is the previous month. Highest scores correspond to better conditions and quality of life;

- the Functioning Assessment Short Test (FAST [14]) in the Italian version [15], a brief instrument designed to assess the main functioning problems experienced by psychiatric patients and by bipolar patients specifically. It includes 24 items assessing impairment or disability in six domains of functioning: autonomy, occupational functioning, cognitive functioning, financial issues, interpersonal relationships and leisure time;

- the HAM-D (Hamilton Rating Scale for Depression) to measure depressive symptoms [16].

\section{STATISTICAL ANALYSIS}

Statistical significance was calculated using $2 \times 2$ tables with Fisher Exact test or $\chi 2$ test. The variables measured by numerical data were compared using the MANOVA statistics for repeated measure or ANOVA 1 way for non-repeated measures.

\section{RESULTS}

Out of the 23 women enrolled, only 18 concluded the study $(78.3 \%)$ and 5 dropped out $(21.7 \%) .7$ out of 18 patients $(38.9 \%)$ concluding the trial followed an antidepressant therapy (5 SNRI and 2 SSRI) (see Table 1). The antidepressant group was senior, but the age difference between the two groups did not reach the statistical difference (Table 1). During the one-year follow-up, antidepressant therapy duration ranged from 6 to 12 months (mean 9.14 \pm 2.79 ). The 
Table 2. Comparing Outcome Measures in the Sample

\begin{tabular}{|c|c|c|c|c|c|c|}
\hline Therapy & $\begin{array}{c}\text { T1 FIQ } \\
\text { Score }(\text { mean } \pm \text { SD })\end{array}$ & $\begin{array}{c}\text { T2FIQ } \\
\text { Score }(\text { mean } \pm \text { SD })\end{array}$ & $\begin{array}{c}\text { SF-12 } \\
\text { Score }(\text { mean } \pm \text { SD })\end{array}$ & $\begin{array}{c}\text { MDQ } \\
\text { Score }(\text { mean } \pm \text { SD })\end{array}$ & $\begin{array}{c}\text { HAM-D } \\
\text { Score }(\text { mean } \pm \text { SD })\end{array}$ & $\begin{array}{c}\text { FAST } \\
\text { Score }(\text { mean } \pm \text { SD })\end{array}$ \\
\hline $\begin{array}{c}\text { Antidepressants } \\
\qquad(N=7)\end{array}$ & $62.42 \pm 20.70$ & $72.11 \pm 13.38$ & $21.85 \pm 3.53$ & $6.43 \pm 3.78$ & $12.28 \pm 5.85$ & $21.71 \pm 8.47$ \\
\hline $\begin{array}{c}\text { No Antidepres- } \\
\text { sants } \\
(N=11)\end{array}$ & $58.65 \pm 21.61$ & $52.82 \pm 15.86$ & $29.36 \pm 3.64$ & $5.36 \pm 3.61$ & $7.00 \pm 3.09$ & $13.54 \pm 8.26$ \\
\hline $\begin{array}{c}\text { Statistics }(d f \\
1,16,17)\end{array}$ & $\begin{array}{c}F=0.092 \\
p=0.766 \\
d f(1,16,17)\end{array}$ & $\begin{array}{c}F=7.095 \\
p=0.017 \\
\operatorname{GL}(1,16,17)\end{array}$ & $\begin{array}{l}F=18.65 \\
p=0.01\end{array}$ & $\begin{array}{l}F=0.363 \\
p=0.555\end{array}$ & $\begin{array}{l}F=6.34 \\
p=0.023\end{array}$ & $\begin{array}{l}F=4.11 \\
p=0.05\end{array}$ \\
\hline
\end{tabular}

patients on antidepressants were all - but one - positive for lifetime prevalence of Depressive Episode at SCID-IV; in the group of patients without antidepressants 6 out 11 had a lifetime Depressive Episode (85.7\% vs. $60 \%$; $p=0.316$, Fisher Exact test). In the antidepressant group 5 had a diagnosis of Major Depressive Disorder against 4 in the control group ( $71.4 \%$ vs. $36.36 \%$; $=0.147$ Fisher Exact Test); one had a diagnosis of Bipolar Disorder against three in the control group ( $14.2 \%$ vs. $27.3 \%$; $=0.429$, Fisher Exact Test); four were positive at MDQ as against four in the control group $(57.1 \%$ vs. $37.7 \%)(\mathrm{p}=0.387$ Fisher Exact Test). Considering the answers at SCID-IV, only 5 patients in the antidepressant group had depression during the follow-up, against 4 in the control group with no differences in the two groups $(71.2 \%$ vs. $36.4 \%$; Fisher Exact test $p=0.335)$. At evaluation at $\mathrm{t} 2,4$ patients in the antidepressant group and 3 in the non-antidepressants group had a current depressive episode, with no statistical difference in the two groups (57.1\% vs. $27.3 \%$; Fisher exact test $\mathrm{p}=0.333$ ). The improvement of depression during the follow-up not differ in the patients who, in both groups, had no current depression: 1 depressed patient out of 5 at follow-up in the antidepressant group, as against 1 out 3 in the control group (20\%vs $33.3 \%$; $\mathrm{p}=0.999$ Fisher Exact test).

The mean and standard deviation of the FIQ score of the two groups (see Table 2) did not show any difference at $\mathrm{t}$, but 12 months later the antidepressant group showed an increase in the mean FIQ scores in opposition to the group without antidepressants; consequently the two group mean scores reached a difference with statistical significance $(72.11 \pm 13.38$ in the antidepressant group as against $52.82 \pm 15.86$ in the non-antidepressant group at $\mathrm{t} 1, \mathrm{~F}=7.09$, $\mathrm{P}=0.017$ at MANOVA 1 way).

At $t 2$ the mean score of SF-12, measuring the quality of life in the two groups, showed a statistically significant difference $(21.85 \pm 3.53$ vs. $29.36 \pm 3.64, \mathrm{P}<0.01$, ANOVA 1 way) with higher scores indicating a better subjective quality of life in the control group. The difference at the FAST score in the two groups, measuring specifically the disability for bipolar symptoms, reaches the threshold of statistical significance.

At the end of the follow up the score at MDQ did not differ in the two groups as did the HAM-D score; the FAST score in the two groups $(21.71 \pm 8.47$ vs. $13.54 \pm 8.26)$ was on the threshold of a statistically significant difference (see table 2).

\section{DISCUSSION}

The study indicates that in a small sample of women with fibromyalgia, after a year of observation, those who had followed a therapy with antidepressants experienced a worst impact of the disease on their daily lives. At the end of the trial the quality of life and the disability linked to bipolar symptoms were worse in the group treated with antidepressants.

The group of patients on the antidepressant therapy did not seem to achieve the expected results even on depressive symptomatology. In fact the number of patients who recovered from a depressive episode during the trial (20\%) did not differ between those taking antidepressants and those who did not $(33.3 \%)$ and this percentage was much lower than expected from the literature on Major Depressive Disorders (40-60\%). Finally the HAM-D score at the end of the trial was surprisingly worse in the group taking antidepressant in comparison to the control group.

How can the worse impact on the disease and the low response to depressive symptoms be explained for the longterm treatment with antidepressants on patients with fibromyalgia?

The cause of the close association of mood disorders with the short-term efficacy of antidepressants in FM patients is not clear yet. Specific polymorphisms of the genes codifying serotonin transporters, and the catechol-O-methyltransferase enzyme that inactivates catecholamine neurotransmitters were both supposed to be implicated in the pathogenesis of mood disorders, and were found in FM [17]. But the efficacy of antidepressants on this disorder may be related to the analgesic effect of antidepressants rather than to their effect on mood symptoms, and appears stronger in agents with mixedreceptor or predominantly noradrenergic activity, rather than on serotoninergic activity [18].

Earlier our group had reported a possible increased risk of bipolar disorder in patients with fibromyalgia [3]. These results were challenged by those who claim that the screening tool we had used - the Mood Disorder Questionnaires was not fit for the purpose [19]. Some later studies confirmed the high risk of Bipolar Disorder in FM patients and 
found that Fibromyalgia subjects who screened positive for bipolar disorder had more severe depression than those with a negative screening. In agreement with our previous survey, the authors reaffirmed that patients with bipolar disorder may experience mood destabilization when treated with duloxetine and milnacipran, commonly used and approved for the treatment of fibromyalgia, and that patients with fibromyalgia should be systematically screened for bipolar disorder prior to treatment [20]. Meanwhile some case reports were published of antidepressants-induced mania in fibromyalgia patients [21]. These observations have become extremely topical thanks to the fact that a recent study [22] has advanced the hypothesis that the premorbid overactivity well described in subjects with fibromyalgia [23] is a core feature of the manic/hypomanic symptoms characterizing bipolar spectrum disorders [24, 25]. A large series of consecutive patients with fibromyalgia was assessed for bipolar spectrum disorders using both a categorical approach (based on a modified version of the DSM-IV SCID-CV interview [10]) and a dimensional approach (based on the screening instrument called hypomania symptom checklist HCL-32 $[26,27])$. Both the "restricted" DSM-IV criteria for Bipolar Disorders and the more inclusive Zurich criteria diagnosed high rates of bipolar spectrum disorder in patients with fibromyalgia ( $70 \%$ and $86.3 \%$, respectively). The authors concluded that the overactivity reported in previous studies may be considered a core feature of the hypomanic symptoms in the syndromes that are comorbid with bipolar spectrum disorders.

Antidepressants are known not to be very effective on bipolar depression or anyhow are less effective on bipolar depression than on depressive episode during the course of a major depressive disorder [28, 29].

In our sample of over $40 \%$ of patients were screened positive for bipolar disorders, and over $20 \%$ had a bipolar disorder diagnosed with the high cut-off of the current DSMIVR (APA 2000) classification [30]. These frequencies do not seem high in comparison with the above-mentioned data, which report $59 \%$ of positive subjects at MDQ in our previous study [3], approximately $30 \%$ of positive screens in the U.S. study [20], and over $70 \%$ of cases diagnosed with the more inclusive Zurich criteria in the study of Alciati et al. [22]. In the light of these considerations the low efficacy of the long-term treatment with antidepressants in fibromyalgia demonstrated by this study is no surprise.

Since it is very difficult to identify bipolar depression and, more generally, to diagnose bipolar disorders [31, 32], the use of antidepressants in a non-psychiatric setting and with non-psychiatric indications in patients at high risk of bipolar disorder, as are the patients with fibromyalgia, could increase the non-recognition of bipolar disorder and, therefore, the inappropriate use of antidepressants. An antidepressant treatment without stabilizers in a bipolar disorder can worsen the course of the illness due to the poor response to the depressive symptoms, and even worsen the mania components $[4,5]$. These negative effects and their consequences on the life of the patient should be evaluated in the long term, because a 12 -week clinical trial is more likely to show positive effects (e.g. on pain) and hide the very negative ones, e.g. inducing irritability. This effect was also seen to occur in patients apparently suffering from Major Depressive Disorder, which was therefore called "false major depressive disorder" [33]. These considerations point to a cautious attitude in the use of antidepressants in a supposed population at high risk of bipolar disorder.

There is another interpretation that could be taken into account to explain the results of the study.

It is possible to suspect that drugs called "antidepressants" can worsen depression, whether unipolar or bipolar. They can be "depressant". This is especially possible in the long run. Favorable initial responses to AD ("the honeymoon with AD") often fade over time. The same drugs that have induced improvements in the beginning, can over time worsen the clinical picture for unknown reasons. The literature on this topic is almost nonexistent bud some authors suggest this hipothesys for possible neurobiological alterations induced by prolonged treatment [34].

\section{LIMITATIONS}

This finding should be discussed taking into account the limitations of the study. This is an observational research, conducted on a few patients and not specifically designed to test the hypothesis. The sample could not be perfectly balanced for variables that might become confounding factors. The results have a heuristic value only. Nevertheless it adds something to what has been demonstrated by randomized controlled trials on the effectiveness of antidepressants. Or, rather, it emphasizes that the debate on the use of antidepressants in fibromyalgia lacks data on their efficacy and safety in the long term.

\section{CONCLUSION}

The results of our study should be read in light of the high prevalence, in our sample, of patients screened positive for Bipolar Disorders; of the literature data indicating a higher risk of bipolar disorder in fibromyalgia, and the poor response of mood symptoms to a treatment with antidepressants in Bipolar Disorders. The deterioration in the long-term management of patients with fibromyalgia following a treatment with antidepressants suggests the need for new and more robust studies on this aspect that was never studied before.

\section{CONFLICT OF INTEREST}

The authors confirm that this article content has no conflicts of interest.

\section{ACKNOWLEDGEMENTS}

Declared none.

\section{REFERENCES}

[1] Goldenberg DL, Burckhardt C, Crofford L. Management of fibromyalgia syndrome. JAMA 2004; 2388-95.

[2] Häuser W, Urrútia G, Tort S, Uçeyler N, Walitt B. Serotonin and noradrenaline reuptake inhibitors (SNRIs) for fibromyalgia syndrome. Cochrane Database Syst Rev 2013; 1: CD010292.

[3] Carta MG, Cardia C, Mannu F, et al. The high frequency of manic symptoms in fibromyalgia does influence the choice of treatment? Clin Pract Epidemol Ment Health 2006; 2: 36. 
[4] Fountoulakis KN, Kasper S, Andreassen O, et al. Efficacy of pharmacotherapy in bipolar disorder: a report by the WPA section on pharmacopsychiatry. Eur Arch Psychiatry Clin Neurosci 2012; 262 Suppl 1: 1-48.

[5] Ei-Mallakh RS, Karippot A. Antidepressant-associated chronic irritable dysphoria (acid) in bipolar disorder: a case series. J Affect Disord 2005; 84(2-3): 267-72.

[6] Carta MG, Balestrieri M, Murru A, Hardoy MC. Adjustment Disorder: epidemiology, diagnosis and treatment. Clin Pract Epidemiol Ment Health 2009; 5: 15.

[7] Wolfe F, Clauw D J, Fitzcharles M-A, et al. The American College of Rheumatology Preliminary Diagnostic Criteria for Fibromyalgia and Measurement of Symptom Severity. Arthritis Care Res 2010; 62 (5): 600-10.

[8] Burckhardt CS, Clark SR, Bennett RM. The fibromyalgia impact questionnaire (FIQ): development and validation. J Rheumatol 1991; 18: 728-33.

[9] Sarzi-Puttini P, Atzeni F, Fiorini T, et al. Validation of an Italian version of the Fibromyalgia Impact Questionnaire (FIQ-I). Clin Exp Rheumatol 2003; 21(4): 459-64.

[10] First MB, Spitzer RL, Gibbon M, Williams JBW. Structured clinical interview for DSM-IV Axis I Disorders clinician version (SCID-CV). Washington DC: American Psychiatric Press 1997.

[11] Hirschfeld RM, Calabrese JR, Weissman MM, et al. Screening for bipolar disorder in the community. J Clin Psychiatry 2003; 64(1): 53-9.

[12] Hardoy MC, Cadeddu M, Murru A, et al. Validation of the Italian version of the "Mood Disorder Questionnaire" for the screening of bipolar disorders. Clin Pract Epidemol Ment Health 2005; 1: 8.

[13] Ware J Jr, Kosinski M, Keller SD. A 12-Item Short-Form Health Survey: construction of scales and preliminary tests of reliability and validity. Med Care 1996; 34(3): 220-33.

[14] Rosa AR, Sánchez-Moreno J, Martínez-Aran A, et al. Validity and reliability of the Functioning Assessment Short Test (FAST) in bipolar disorder. Clin Pract Epidemiol Ment Health 2007; 3: 5.

[15] Moro MF, Colom F, Floris F, et al. Validity and Reliability of the Italian Version of the Functioning Assessment Short Test (FAST) in Bipolar Disorder. Clin Pract Epidemiol Ment Health 2012; 8: 6773.

[16] Hamilton M. A rating scale for depression. J Neurol Neurosurg Psychiatry $1960 ; 23: 56-62$.

[17] Van Houdenhove B, Egle UT. Fibromyalgia: a stress disorder? Piecing the biopsychosocial puzzle together. Psychother Psychosom 2004; 73: 267-75.

[18] Maizels M, McCarberg B. Antidepressants and antiepileptic drugs for chronic non-cancer pain. Am Fam Physician 2005; 71: 483-90.

[19] Zimmerman M, Galione JN, Chelminski I, Young D, Dalrymple K. Psychiatric diagnoses in patients who screen positive on the Mood Disorder Questionnaire: Implications for using the scale as a case-finding instrument for bipolar disorder. Psychiatry Res 2011; 185(3): 444-9.

[20] Wilke WS, Gota CE, Muzina DJ. Fibromyalgia and bipolar disorder: a potential problem? Bipolar Disord 2010; 12(5): 514-20.

[21] Mustafa AF, Almoshmosh N, Al-Robb H, AbuKmeil S. A Case of Possible Duloxetine-Induced Mania, German J Psychiatry 2010. Available at: http://www.gjpsy.uni-goettingen.de

[22] Alciati A, Sarzi-Puttini P, Batticciotto A, et al. Overactive lifestyle in patients with fibromyalgia as a core feature of bipolar spectrum disorder. Clin Exp Rheumatol 2012; 30(SUPPL.74): S122-8.

[23] Van Houdenhove B, Neerinckx E, Onghena P, Lysens R, Vertommen H. Premorbid "overactive" lifestyle in chronic fatigue syndrome and fibromyalgia: An etiological factor or proof of good citizenship? J Psychosom Res 2001; 51(4): 571-6.

[24] Carta MG, Angst J. Epidemiological and clinical aspects of bipolar disorders: controversies or a common need to redefine the aims and methodological aspects of surveys. Clin Pract Epidemol Ment Health 2005; 1(1): 4.

[25] Akiskal HS. The bipolar spectrum: new concepts in classification and diagnosis. In: Grinspoon L, Ed. Psychiatry update: The American Psychiatry Association Annual Review Washington DC: American Psychiatry Press 1983; pp. 271.92.

[26] Angst J, Meyer TD, Adolfsson R, et al. Hypomania: a transcultural perspective. World Psychiatry 2010; 9(1): 41-9.

[27] Carta MG, Hardoy MC, Cadeddu M, et al. The accuracy of the Italian version of the Hypomania Checklist (HCL-32) for the screening of bipolar disorders and comparison with the Mood Disorder Questionnaire (MDQ) in a clinical sample. Clin Pract Epidemol Ment Health 2006; 8: 2.

[28] Cerullo MA, Strakowski SM. A systematic review of the evidence for the treatment of acute depression in bipolar I disorder. CNS Spectr 2013; 18: 1-10.

[29] Grunze H, Vieta E, Goodwin GM, et al. The world federation of societies of biological psychiatry (WFSBP) guidelines for the biological treatment of bipolar disorders: Update 2012 on the longterm treatment of bipolar disorder. World J Biol Psychiatry 2013; 14(3): 154 - 219 .

[30] American Psychiatric Association. DSM-IV TR. US: APA Washington 2000.

[31] Carta MG, Tondo L, Balestrieri M, et al. Sub-threshold depression and antidepressants use in a community sample: searching anxiety and finding bipolar disorder. BMC Psychiatry 2011; 11: 164.

[32] Carta MG, Aguglia E, Bocchetta A, et al. The Use of Antidepressant Drugs and the Lifetime Prevalence of Major Depressive Disorders in Italy. Clin Pract Epidemiol Ment Health 2010; 6: 94-100

[33] Koukopoulos A, Koukopoulos A. Agitated depression as a mixed state and the problem of melancholia. Psychiatr Clin North Am 1999; 22 (3): 547-64.

[34] Raja M. Delayed loss of efficacy and depressogenic action of antidepressants. J Clin Psychopharmacol 2009; 29(6): 612-4.

\footnotetext{
Received: April 13, 2013

Revised: May 18, 2013

Accepted: May 19, 2013

(C) Carta et al.; Licensee Bentham Open.

This is an open access article licensed under the terms of the Creative Commons Attribution Non-Commercial License (http://creativecommons.org/licenses/by-nc/3.0/) which permits unrestricted, non-commercial use, distribution and reproduction in any medium, provided the work is properly cited.
} 